\title{
Una breve Comunicación desde Irán sobre el consumo de tabaco entre los estudiantes de Medicina, los Médicos Residentes y los Médicos Adjuntos
}

\author{
Jamshid Ahmadi, MD, Profesor \\ de Psiquiatría \\ Hosein Khalili, MD \\ Reza Jooybar, MD \\ Nooreddin Namazi, MD \\ Pedram Mohammad Aghaei, MD, \\ Residente de Psiquiatría \\ Hospital de Hafez, Shiraz \\ IRÁN
}

\begin{abstract}
RESUMEN - Objetivo: Evaluar la prevalencia de fumadores entre los estudiantes de Medicina iraníes, Médicos Residentes y Adjuntos.

Método: Los sujetos (532 varones y mujeres) se seleccionaron al azar en la Universidad de Shiraz de Ciencias Médicas y a todos ellos se les pasó una encuesta.

Resultados: De acuerdo con los resultados obtenidos un $16,79 \%$ de varones y $0,69 \%$ de mujeres eran fumadores. Entre los estudiantes de Medicina, encontramos que el 18,48\%. de los estudiantes de primer año,el 12,5\% de los de segundo año,el 7,19\% de tercer año y el 16,95\% de los estudiantes internos eran fumadores. En cambio,entre los Médicos Residentes sólo el 11,11\% eran fumadores y el 7,57\% de los Médicos Adjuntos fumaban. Las causas más habituales para fumar eran la necesidad de evitar los síntomas tras la retirada, el placer que supone, y por último como forma de eliminar la tensión. El tabaco con filtro era el que más se consumía. La media de cigarrillos por día era de 6,29 y la edad más frecuente de inicio eran los 19,71 años.
\end{abstract}

\section{Introducción}

La dependencia a la nicotina constituye un trastorno psiquiátrico (DSM-IV, Asociación Psiquiátrica Americana 1994), y la tasa de prevalencia de la dependencia a la nicotina basada en criterios diagnósticos es de un $20 \%$, siendo del $24 \%$ entre la población general (Breslau et al. 1991, Anthony et al. 1994). Pero existen tasas muy dispares según el sesgo. 
Las tasas de prevalencia de dependencia a nicotina en Hong Kong son del $27 \%$ en el hombre y del $1 \%$ en la mujer (Chen et al. 1993), y en Shanghai del $39 \%$ y del $2 \%$, respectivamente (Lee et al. 1990).

La dependencia a la nicotina es más frecuente entre los hombres (Lee et al. 1990, Chen et al. 1993, Ahmadi J. \& Ghanizadeh À. 1999) y entre las personas con ingresos más bajos (Anthony el al. 1994) y con peor nivel educativo (Breslau et al. 1991, Anthony et al. 1994).

En Irán existe actualmente una gran preocupación entre los investigadores y los políticos por la mayor incidencia del consumo de drogas psicoactivas entre la población universitaria, pero hay poca información sobre el consumo de drogas en este país. El uso del alcohol está prohibido tanto legal como religiosamente, al igual que el consumo de otras drogas salvo en el caso del tabaco para el que no existe ninguna prohibición.

Anteriormente la tasa de fumadores entre los estudiantes de Medicina iraníes del sexo masculino (fumadores habituales) era del 11,5\% (Ehsanmanesh M. 1996) y del 6,3\% (Borhanihaghighi, UN 1995).

El objetivo de este estudio es el de evaluar la proporción de fumadores entre los estudiantes de Medicina, Médicos Residentes y Médicos Adjuntos de la Universidad de Ciencias Médicas de Shiraz en Irán, y para ello se realizaron las siguientes preguntas: 1. ¿Cuánto fuma? 2. ¿Cuál es el motivo por el que fuma?, 3. ¿Qué cantidad de tabaco fuma diariamente?, 4. ¿A qué edad se inicia en el consumo de tabaco?

\section{Material y método}

\section{Sujeto}

Se trataba de 532 participantes seleccionados al azar entre los 2.128 estudiantes de Medicina, Residentes y Médicos Adjuntos. De los 368 estudiantes de primer año se escogió a 92, y de los 288 de segundo año a 72. Esta selección aleatoria se realizó también en estudiantes de Tercer y Cuarto año obteniendo una selección de 139 y 118 de los 556 y 472 estudiantes de dichos años respectivamente. Por otro lado, de entre los 180 Médicos Residentes, se seleccionó a 45 y finalmente a 66 de los 264 Médicos Adjuntos existentes.

\section{Instrumento}

Se utilizó una encuesta con el fin de evaluar la proporción de fumadores. Se incluyeron en ella varias preguntas referidas a edad, sexo, año de carrera, si fumaban en ese momento, número de cigarrillos al día, edad de inicio, motivo por el que se iniciaron en el consumo y razón por la que lo mantienen.

\section{Procedimiento}

Tras reunir a los participantes en una sesión y darles una breve explicación sobre el formulario y la confidencialidad del mismo, se distribuyó un cuestionario de elección múltiple basado en estudios anteriores. Los encuestados tuvieron 10 minutos para completar dicho cuestionario. 


\section{Resultados}

En el caso de los hombres (387) 65 $(16,49 \%)$ eran fumadores frente a 322 $(83,21 \%)$ que no lo eran. Entre las mujeres (145) sólo el 0,69\% fumaba. El 91\% del total fumaba tabaco extranjero. La edad media de inicio en el tabaco fue de 19,7 años con una media de 6,29 cigarrillos/día. La tabla I muestra el número de fumadores según los distintos grupos estudiados. El 12,4\% del total eran fumadores. La tabla II nos presenta los motivos por los que se fuma, observándose como el $33,3 \%$ lo hace como consecuencia de los síntomas que padecieron tras intentar abandonar el tabaco.

\section{Discusión}

Según el presente estudio, el consumo de tabaco está significativamente relacionado con el sexo masculino. Esto también contrasta en una investigación llevada a cabo en los Estados Unidos que muestra variaciones significativas en relación con el sexo (Maddux J.F,. Hoppe S.K. \& Gostello R.M. 1986). Por otro lado un estudio realizado en Irán hace algunos años mostró cómo el empleo del tabaco se acompañaba del consumo de otras sustancias. Así, el $43,7 \%$ de los individuos que fumaban, también tomaban otro tipo de sustancias. Entre las personas que no fumaban, sólo el

Tabla I

Porcentaje de fumadores y no fumadores en diferentes grupos

\begin{tabular}{|c|c|c|c|c|c|}
\hline & \multicolumn{2}{|c|}{ No fumadores } & \multicolumn{2}{|c|}{ Fumadores } & \multirow[b]{2}{*}{ Total } \\
\hline & $\mathrm{N}$ & $\%$ & $\mathrm{~N}$ & $\%$ & \\
\hline Estudiantes de $1^{\text {er }}$ año & 17 & 18,48 & 75 & 81,52 & 92 \\
\hline Estudiantes de $2^{\circ}$ año & 9 & 12,5 & 63 & 87,5 & 72 \\
\hline Estudiantes de $3^{\text {er }}$ año & 10 & 7,19 & 129 & 92,81 & 139 \\
\hline Estudiantes de $4^{\circ}$ año & 20 & 16,95 & 98 & 83,05 & 118 \\
\hline Médicos Residentes & 5 & 11,11 & 40 & 88,89 & 45 \\
\hline Médicos Adjuntos & 5 & 7,57 & 61 & 92,43 & 66 \\
\hline Total & 66 & & 406 & & 532 \\
\hline
\end{tabular}

Tabla II

Causa /razón para fumar

\begin{tabular}{lrc} 
Razón & N & $\%$ \\
\hline Necesidad (evitar los síntomas de abstinencia) & 22 & 33,3 \\
Producir placer & 20 & 30,3 \\
Relajar la tensión & 16 & 24,2 \\
Otras & 8 & 12,1 \\
Total & 66 & 100 \\
\hline
\end{tabular}


$14,2 \%$ utilizaban otro tipo de drogas (Ahmadi J. \& Ghanizadeh À. 1999). El tabaco en nuestra investigación aparece como la sustancia más utilizada entre los estudiantes de Medicina iraníes, frente al alcohol que es la más empleada por los estudiantes de Medicina occidentales. (Kory W.P. \& Crandall L.A. 1984).

Además el 33,3\% de los individuos señalan como era la necesidad de evitar los síntomas de dependencia la causa más común para el mantenimiento del consumo del tabaco mientras que la razón fundamental de su inicio fue la de aliviar la tensión psicológica.

Por otro lado la proporción más alta de tabaquismo se daba durante el primer año de carrera $(18,42 \%)$, mientras que la proporción más baja la ofrecían las Médicos Adjuntos (7,57\%). En nuestro estudio, el $16,79 \%$ de los hombres y el $0,69 \%$ de las mujeres eran fumadores de tabaco, mientras que en otro estudio lo eran el $26 \%$ de los hombres iraníes y el 3,8\% de las mujeres, (Ahmadi, J. Khalili, H. Hooybar, R. Namazi, N. \& Mohammadaghaee, P. 1998).

\section{Bibliografía}

AHMADI, J., GHANIZADEH, A. Psychoactive substance use among Iranian medical students. Unpublished, 1999.

AHMADI, J.,KHALILI,H.,JOOYBAR,R., NAMAZI, N. MOHAMMADAGHAEE, P. Epidemiology of cigarette smoking in Iran. Presented at fifth Iranian Congress of
Research in Psychiatry and Psychology. Teheran, Iran, Feb. 28-Mar. 3, 1998.

American Psychiatry Association Diagnostic and Statistical Manual of Mental Disorders, 4 th edition (Washington DC, American Psychiatric Association), 1994.

ANTHONY, J.C., WARNER, L.A., KESSLER, R. Comparative epidemiology dependence on tobacco, alcohol, controlled substances and inhalants: basic findings from the National Comorbidity survey, Experimental and clinical psychopharmacology, 2, 244-268, 1994.

BORHANI HAGHIGHI, A. Relationship between anxiety and cigarette smoking in male medical students in Shiraz-IRAN, Thesis, unpublished, 1995.

BRESLAU, N., KILBEY, M.M.,ANDRESKI, P. Nicotine dependence, major depression, and anxiety in young adults. Archives of General Psychiatry, 48, 1069-1074, 1991.

CHEN, C.N., WONG. J., LEE, N., CHAN-HO, M.W., LAU, J.T., FUNG, M. The Shatin community mental health survey in Hong Kong II. major findings, Archives of General Psychiatry, 50, 125-133, 1993

EHSANMANESH, M. Rate and causes of cigarette smoking among Shiraz male medical students, Shiraz-Iran, Thesis. Unpublished, 1996.

KORY, W.P.,CRANDALL,L.A. Non- medical drug use patterns among medical students. The International Jour nal of Addiction, 19 (8) 871-884, 1984.

LEE, C.K., KWAK, Y.S., YAMAMOTO, J. et al. Psychiatric epidemiology in Korea part I: general and age differences in Seoul, Journal of Nervous and Mental Disor ders, 178, 242-246, 1990.

MADDUX, J.F., HOPPE, S.K., GOSTELLO, R.M. Psychoactive substances use among medical students. American Journal of Psychiatry, 143: 187-191, 1986.

Dirección para correspondencia:

Jamshid Ahmadi

P.O. Box: 71345 - 1416

Hospital de Hafez

Shiraz

IRÁN 